\title{
Forensic Facial Reconstruction Using 3D Printing
}

\author{
NICOLETA-VIOLETA STANCIU ${ }^{*}$, RAZVAN-TUDOR ROSCULET ${ }^{\mathbf{1}}$, CATALIN FETECAU ${ }^{1}$, \\ COSTEL TAPU ${ }^{2}$ \\ ${ }^{1}$ Center of Excellence in Polymer Processing, Dunarea de Jos University of Galati, 47 Domneasca, 800008, Galati, Romania \\ ${ }^{2}$ Galati County Police Inspectorate - Forensic Department, 200 Brailei, 800408, Galati, Romania
}

\begin{abstract}
The paper presents the application of $3 D$ printing in the forensic field in order to perform facial reconstruction on a $3 D$ printed replica of the victim's skull. Firstly, imagine data from a computed tomography of a skull was converted into a 3D model. Then, the 3D skull model was sliced and printed in different positions in order to optimize the $3 D$ printing configuration. Since the quality of the $3 D$ printing process depends on the thermal and rheological properties of the $3 D$ printing filaments, the rheological behavior of the ABS was investigated using melt flow rate and capillary rheometry. Lastly, an accurate skull replica was achieved using the optimal printing parameters. The $3 D$ printed skull was used to perform the facial reconstruction of the victim by the forensic team. Based on the results of the present research, the $3 D$ printing technology is a feasible solution to obtain anatomically accurate skull replicas.
\end{abstract}

Keywords: 3D printing, slicing optimization, facial reconstruction, ABS, melt flow rate, shear viscosity

\section{Introduction}

When typical identification methods, such as DNA, dental, and radiography data, are unavailable in order to identify the deceased person, the morphology of the face is approximated by facial reconstruction [1-3]. Since the facial soft-tissues depth varies for different ethnic groups and regions of the face, generally, the average thickness of the soft tissues is used for reconstruction [2-5]. For many years, facial reconstruction was performed directly on the skull, but having soft-tissue thickness variations which can recreate several types of morphologies, the preferred reconstruction methods involved computer-based systems and replicas of the skull $[1,2,6]$.

Additive manufacturing (AM), or 3D printing, has rapidly advanced in the last decade and still gains growing interest. AM is fundamentally different from traditional manufacturing; a structure can be built into its designed part shape using a 'layer-by-layer' approach rather than using formative or subtractive manufacturing technologies. AM is versatile and highly customizable and enables the user to manufacture complex geometries and lighter structures with low plastic waste, energy and cost. Therefore, various industries, such as automotive [7-9], aerospace [7-9], healthcare [7, 8, 10-12] and constructions [12], produce complex parts using a wide range of materials (metals, few polymers, biomaterial, ceramics). For the past years, medical applications are developing rapidly by using the 3D printing technology [13] in areas ranging from creating organs and tissues to patient-specific models, such as custom-made prosthetics. Another use of the 3D printing in the medical field is for educational purpose, for example, students can study the human anatomy, prepare for surgeries $[11,13,14]$, and even help with the facial reconstruction process.

Regarding the reconstruction process, the skull replicas used in the forensic field are made by a manual casting process using gypsum $[15,16]$. A drawback of the casting method is that the original skull can be damaged in the handling process, which can be a major issue if it is forensic evidence. A novel method to replicate a victim skull is $3 \mathrm{D}$ printing, which can be done remotely since only a $3 \mathrm{D}$ model is needed [17]. Comparing the surface quality of the cast replica with the printed skull, the gypsum skull is superior to the printed replica, mainly due to the material used in the process $[15,16]$. However, the detailed features (brain and eyes cavities, denture geometry) of the printed skull are far superior to the gypsum skull [17].

*email: nicoleta.stanciu@ugal.ro 
In this paper, we present the applicability of 3D printing to the forensic field by making accurate skull replicas using a scanned model of the original skull.

\section{Materials and methods}

The steps that outline the strategy of the present research paper are presented in Figure 1. The 3D model of the skull (Figure 2) in the form of a Stereo Lithography (STL) file was generated by an X-ray computed tomography (CT scan) by scanning the skull with a layer thickness of 1-mm. In order to reduce the material consumption and printing time and to print skull replicas with minimal defects, the print slicing process was optimized using the Ultimaker Cura software.

The rheological properties are very important in order to optimize the processing temperatures in the manufacturing processes, such as extrusion and 3D printing. A rheological measure widely used in the industry is the melt flow index, which indicates the fluidity of the tested polymer [18]. However, the melt flow index measurements are performed at lower shear rates than the shear rate range of interest in $3 \mathrm{D}$ printing process, which are 50-1000 s-1. Therefore, capillary rheometry measurements are needed in order to asses the flow behavior of the polymer at shear rates.

From the rheological data and the optimization of the slicing process, an optimal skull was printed at 1:1 scale in order to perform the facial reconstruction.

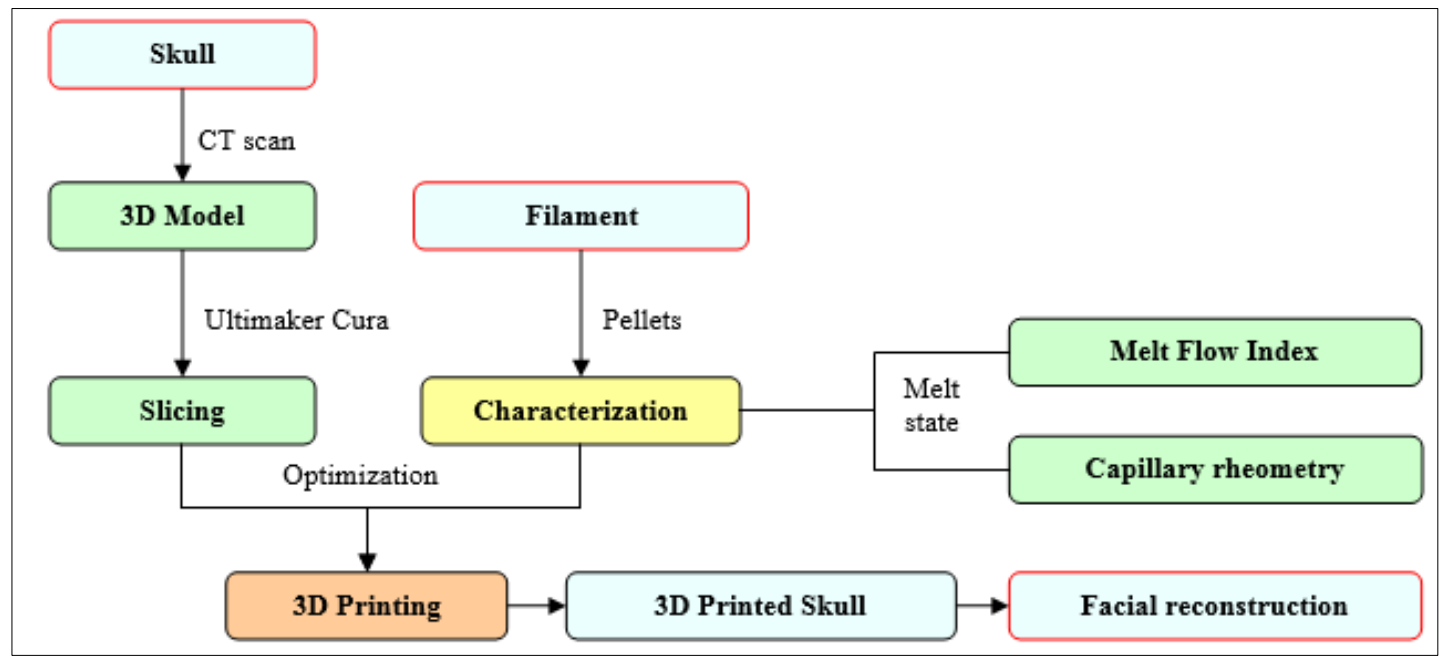

Figure 1. Summary of the research methodology

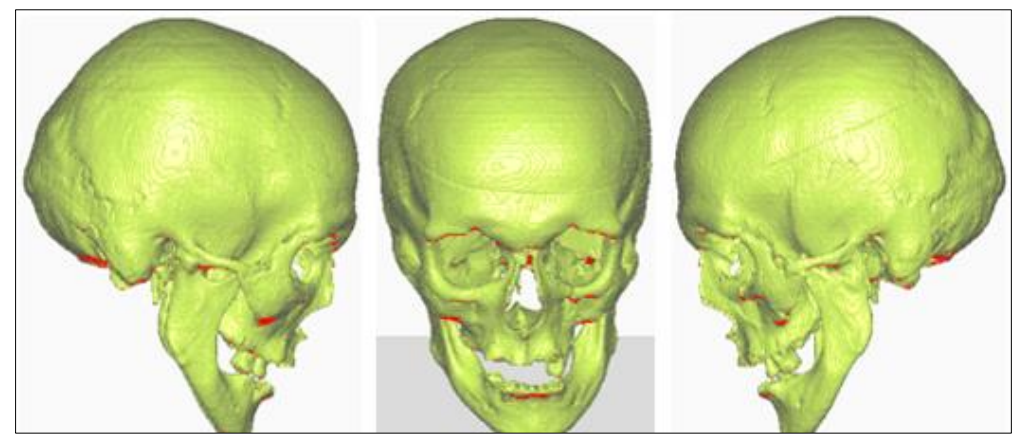

Figure 2. The 3D model of the victim's skull

\subsection{Materials}

The acrylonitrile butadiene styrene (ABS) filament (Verbatim, Eschborn, Germany) with a 1.75-mm diameter was selected due to its high rigidity, resistance to grease [19] and offers a high degree of surface quality. As an impact-resistant engineering thermoplastic, ABS often is suitable in applications for 
automotive, home appliances, electronics, transportation industries and much more, since is easy to process and has a reasonable price-cost effectiveness. Regarding the ABS filament, it's the second most used filament after polyactic acid (PLA) filament since its most used for "wear and tear" prints and the ability to process its surface with acetone and can be painted [20]. However, the printing of ABS has several drawbacks, such as warping, foul odors, it needs to be printed in an enclosed 3D printer in order to minimize the cracks and split layers and is not biodegradable [20].

\subsection{Characterization methods}

The ABS filament was manually cut in pellets with 2 to $3-\mathrm{mm}$ in length to determine the melt flow index of the ABS using a Ceast Melt Flow Quick Indexer (Instron, USA). The measurements were taken according to ISO 1133 , at 215,230 , and $245^{\circ} \mathrm{C}$ melt temperatures and for $1.2,2.16,3.8,5$, and $10 \mathrm{~kg}$ weights. The mean values of five measurements are reported.

In addition, the melt flow behavior of ABS was investigated under the capillary shear flow using a high-pressure capillary rheometer (Rheograph 75, Göttfert $\mathrm{GmbH}$, Buchen, Germany) with a round capillary die with length/diameter $(L / D)$ ratio of 20/1. Before the rheological tests were performed, the material was dried at $80^{\circ} \mathrm{C}$ for $4 \mathrm{~h}$ in a vacuum oven (EV-50, Raypa, Terrassa, Spain). The rheological measurements were carried out for the temperatures of 215,230 , and $245^{\circ} \mathrm{C}$ and shear rate range of 10 $5000 \mathrm{~s}^{-1}$, in decreasing order. The mean values of three measurements are reported.

The rheological data were fitted to the Cross model [18] using the WinRHEO II software (Göttfert GmbH, Buchen, Germany).

$$
\eta(\dot{\gamma})=\frac{\eta_{0}}{1+\left(\frac{\eta_{0} \cdot \dot{\gamma}}{\tau^{*}}\right)^{1-n}} \mathrm{~Pa} \cdot \mathrm{s},
$$

where $\eta_{0}$ is the zero-shear rate viscosity, in $(\mathrm{Pa} \cdot \mathrm{s}), \tau^{*}$ is the critical shear stress where shear thinning behavior starts and $n$ is the power-law index describing the shear-thinning behavior.

\subsection{D Modeling and slicing optimization}

The Ultimaker Cura software (Ultimaker B.V., Utrecht, Netherlands) was used for slicing the 3D model with a layer thickness of $0.2-\mathrm{mm}$, at 1:1 scale. Firstly, the model was sliced in the default position (Figure 3a). Since the default position (Figure 3a) in the slicing software is not optimal due to the small contact surface between the 3D model and building platform, the model was rotated by $135^{\circ}$, as shown in Figures 3b. The 3D model has a complex geometry; therefore, the slice code was generated with the aid of a raft-type adhesion platform, which helps stabilize the model and minimize the warping of ABS. The face-upward position (Figure 3b) of the 3D model was selected to be sliced and printed so that the facial bones, which are more complex in design, would be printed on a larger contact surface (marked "A" in Figure 3b) for a good quality print.

\subsection{D Printing of the skull model}

An in-house design 3D printer (Figure 4) and an ABS filament were selected in order to print the skull by fused deposition modeling (FDM). The sliced 3D model was printed using a 0.4-mm nozzle and the printing parameters: $0.2-\mathrm{mm}$ layer thickness, $245^{\circ} \mathrm{C}$ melt temperature, $75^{\circ} \mathrm{C}$ platform temperature, $40 \mathrm{~mm} / \mathrm{s}$ printing speed, grid infill pattern and $20 \%$ infill density. Since the contact surface of the skull model in the face-upward position is not planar, the model was printed using a raft-type adhesion platform to sustain the entire print. 


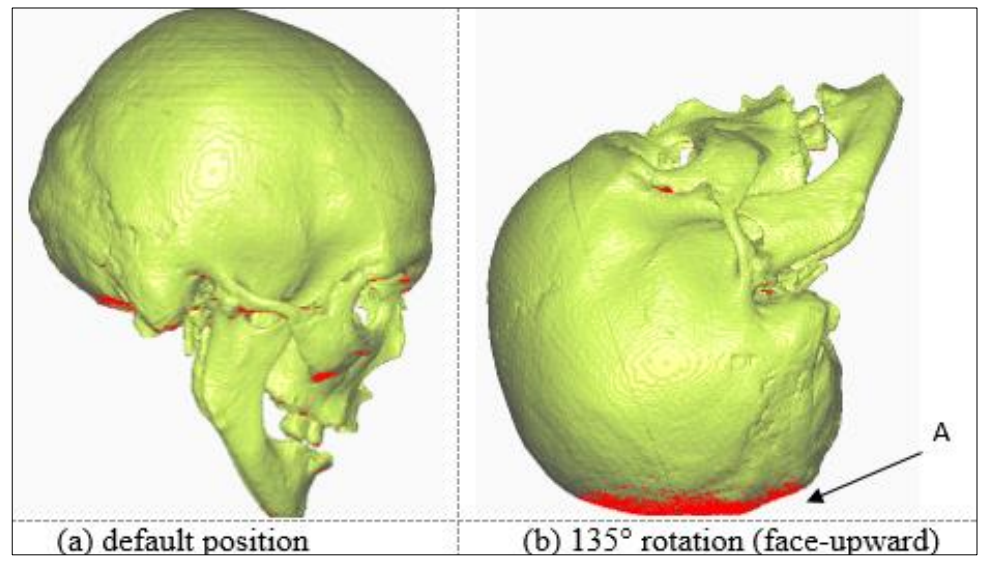

Figure 3. Position of the 3D model for the slicing process

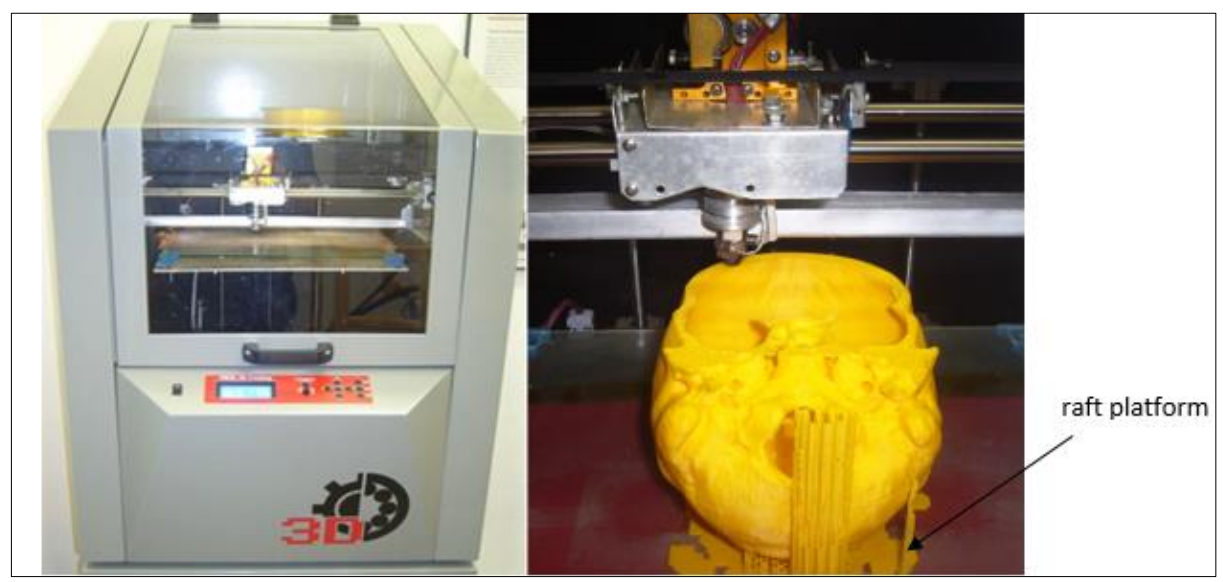

Figure 4. 3D printing of the 1:1 scale model using an in-house design 3D printer

\section{Results and discussions}

\subsection{Melt flow rate}

The melt flow rate (MFR) as a function of temperature and weight for the ABS is represented in Figure 5. The MFR values increases with increasing weight and melt temperature due to an increase in polymer chain mobility and flexibility [21], thus low viscosity and good processability can be achieved by increasing melt temperature. Based on the MFR results, the printing temperature, which is an important 3D printing parameter, can be chosen between 230 and $245^{\circ} \mathrm{C}$ since high values of MFR are needed for the $3 \mathrm{D}$ printing process [21].

\subsection{Melt shear viscosity}

During the rheological measurements performed on ABS, no flow instability occurred and a smooth stream of ABS was produced at the exit of the capillary die. The rheological results in a log-log scale are shown in Figure 6, where the apparent shear viscosity of ABS is reported as a function of apparent shear rate and temperature. It can be observed that the shear viscosity of the ABS decreases with increasing temperature and shear rate, respectively. At the temperatures investigated, the rheological response of the ABS is described by the shear-thinning behavior at shear rates higher than $50 \mathrm{~s}^{-1}$. Since the 50-1000 $\mathrm{s}^{-1}$ shear rate range is of interest for the 3D printing process, the shear-thinning behavior of $\mathrm{ABS}$ corresponds to good 3D printability. 

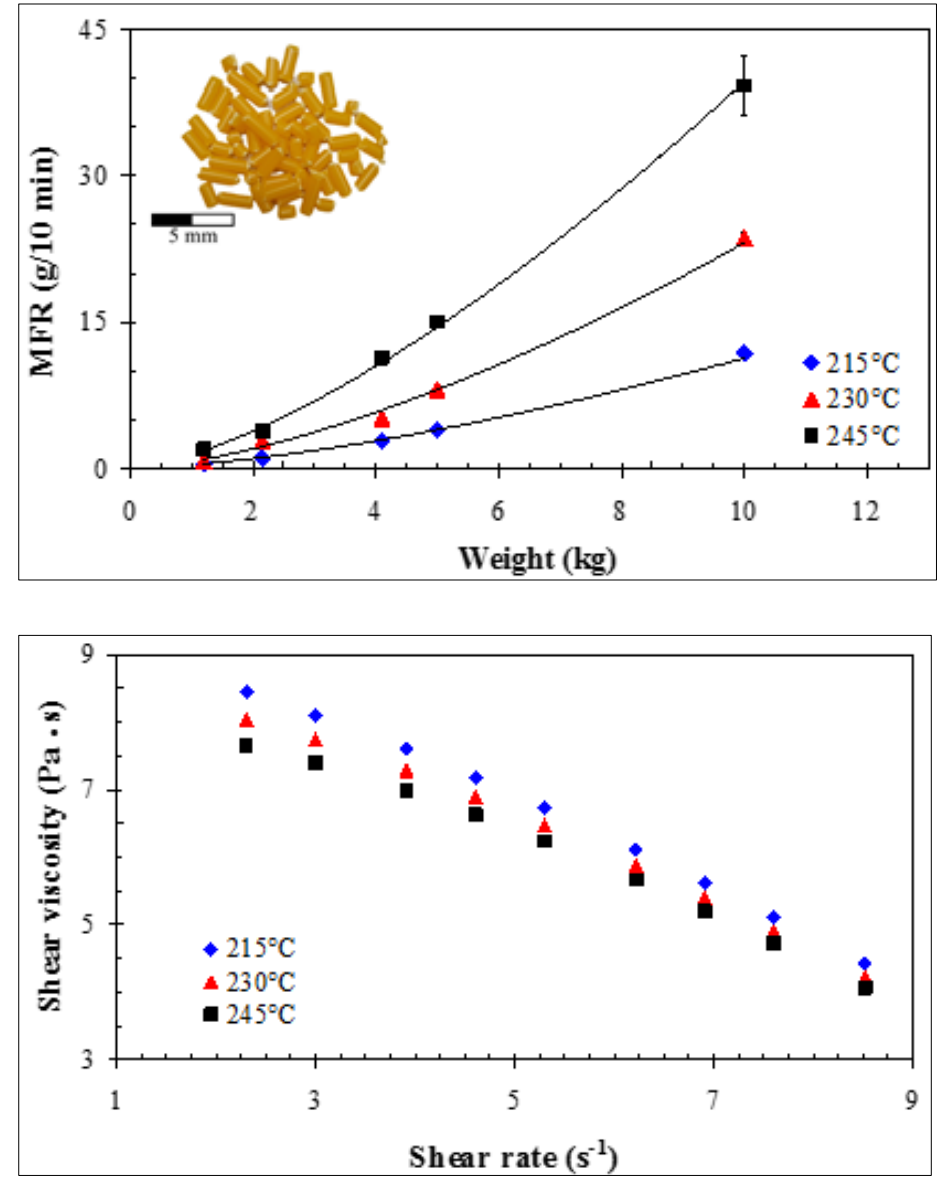

Figure 5. Melt flow rate of ABS

Figure 6. Apparent viscosity of ABS as a function of apparent shear rate and temperature

In addition to the shear-thinning behavior, the activation energy for flow is another parameter that must be considered for printability since it reflects the sensitivity of viscosity to temperature. Generally, in order to have good weldability between the printed layers, low activation energy is required [8]. The temperature dependence of the melt shear viscosity of the ABS is governed by the Arrhenius law, as can be seen in Figure 7 for different shear rates. From the slope of the linear fits, $E_{a} / R_{g}$, the flow activation energy, $E_{a}$, is calculated by replacing the gas constant value, $R_{g}=8.314 \cdot 10^{-3}(\mathrm{~kJ} / \mathrm{mol} \cdot \mathrm{K})$ and is presented in Table 1 . The values of $E_{a}$ are strongly dependent on the shear rate, which decrease with increasing shear rate from 10 to $5000 \mathrm{~s}^{-1}$, suggesting a change in the polymer structure. From the $E_{a}$ values it is expected that the rheology-based weldability of the polymer to be better when shear rates between 200 to $1000 \mathrm{~s}^{-1}$ are in order for 3D printing process.

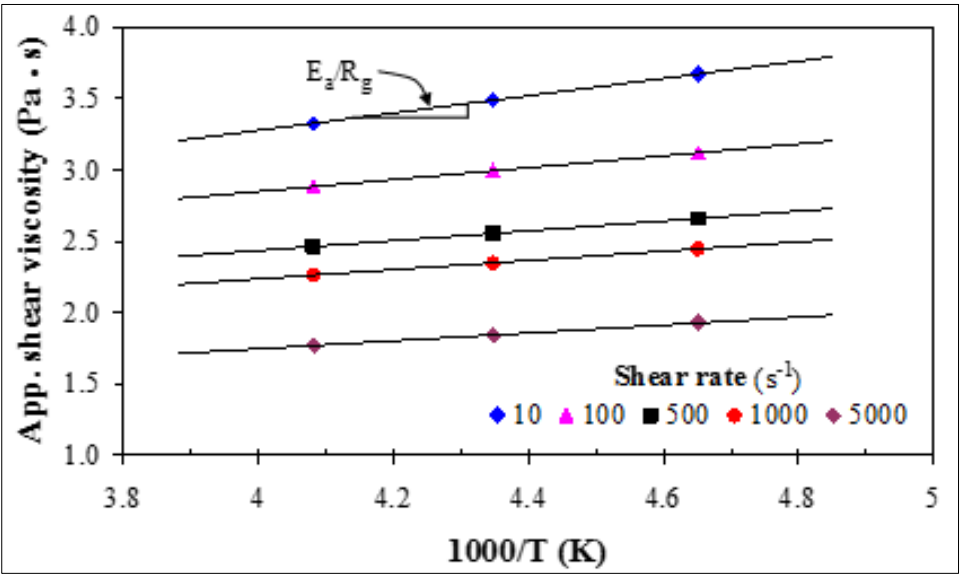

Figure 7. Dependence of shear viscosity on temperature and apparent shear rate $(L / D=20 / 1)$ 
Table 1. Activation energy based on Arrhenius equation

\begin{tabular}{|c|c|c|}
\hline $\begin{array}{c}\text { Shear rate, } \\
\gamma,\left(\mathrm{s}^{-1}\right)\end{array}$ & $\begin{array}{c}\text { Activation energy, } \\
E_{a},(\mathrm{~kJ} / \mathrm{mol})\end{array}$ & \multirow{2}{*}{$R^{2}$} \\
\hline 10 & 5.052 & \multirow{2}{*}{1.000} \\
\hline 20 & 4.543 & \\
\hline 50 & 3.899 & \\
\hline 100 & 3.467 & \\
\hline 200 & 3.130 & \multirow{2}{*}{0.999} \\
\hline 500 & 2.879 & \\
\hline 1000 & 2.697 & \\
\hline 2000 & 2.483 & \\
\hline 5000 & 2.309 & \\
\hline
\end{tabular}

The rheological data of ABS were fitted to the Cross viscosity model for the 215,230 , and $245^{\circ} \mathrm{C}$ melt temperatures, which are shown in Figure 8. The Cross model (solid line) predicts very well the viscosity response of $\mathrm{ABS}$ at the experimental conditions. However, the rheological data do not cover a lower shear rates $\left(<10 \mathrm{~s}^{-1}\right)$ to confidently develop predictions for a Newtonian plateau. At shear rates higher than $50 \mathrm{~s}^{-1}$, the non-Newtonian (shear-thinning) behavior is more pronounced due to polymer chains that align along the flow direction.

The Cross model parameters, the zero-shear viscosity, $\eta_{0}$, the shear stress, $\tau^{*}$, and the shear-thinning index, $n$, are given in Table 2 . The $R^{2}$ values demonstrate a good fitting of the Cross model to experimental rheological data. As shown in Table 2, the ABS exhibits a pronounced solid-like behavior, with an increasing shear-thinning index from 0.281 to 0.312 when the temperature increased from 215 to $245^{\circ} \mathrm{C}$.

\subsection{D printed skull}

Figure 9 shows the 3D printed skull (1:1 scale) using an ABS filament and an in-house design 3D printer. Recall that the skull model was printed in the face-upward position. The 1:1 scale model was printed using $418 \mathrm{~g}$ of ABS filament in a period of time of $38 \mathrm{~h}$. The printed skull is an accurate representation of the original skull with at-scaled anatomically features, such as: cavities of the eyes and brain, bone structures and denture.

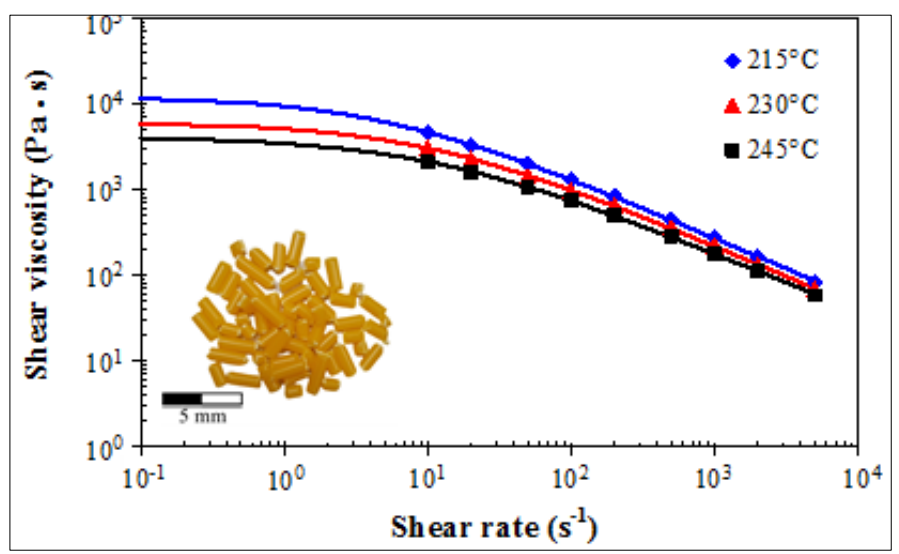

Figure 8. Rheological data (symbols) and Cross model fit (solid lines) for ABS $(L / D=20 / 1)$ 
Table 2. Cross model parameters for ABS

\begin{tabular}{|c|c|c|c|}
\hline \multirow{2}{*}{ Parameter } & \multicolumn{3}{|c|}{ Temperature, } \\
& $\mathbf{2 1 5}\left({ }^{\circ} \mathrm{C}\right)$ & $\mathbf{2 4 5}$ \\
\cline { 2 - 4 } & $12384.496 \pm 189.895$ & $6172.826 \pm 58.082$ & $4171.709 \pm 62.734$ \\
\hline$\eta_{0}(\mathrm{~Pa} \mathrm{~s})$ & $63.414 \pm 0.697$ & $61.376 \pm 0.519$ & $45.717 \pm 0.462$ \\
\hline$\tau^{*}(\mathrm{kPa})$ & $0.195 \pm 0.001$ & $0.101 \pm 0.000$ & $0.091 \pm 0.000$ \\
\hline$\lambda(\mathrm{s})$ & $0.281 \pm 0.002$ & $0.283 \pm 0.000$ & $0.312 \pm 0.001$ \\
\hline$n$ & 1.000 & 1.000 & 1.000 \\
\hline$R^{2}$ & \multicolumn{3}{|c|}{} \\
\hline
\end{tabular}

The quality of the printed skull was investigated for printing defects using a Nikon digital camera (Figure 9). Minor layer delaminations on the outer contour are observed in the printed skull because the large chamber of the printer is not sealed, thus the variation of the chamber's temperature affected the adhesion between the layers. Because the printing was performed using a 0.2-mm layer thickness, the surface roughness of the printed skull is approximately $0.2-\mathrm{mm}$. However, the $0.2-\mathrm{mm}$ roughness can be seen as an advantage since the clay, used in the following stages of the reconstruction, needs to adhere to a rough surface. However, to improve the quality of the 3D printed surface, the CT scan of the skull could be performed at a slicing thickness of less than 1-mm based on the previous observation.

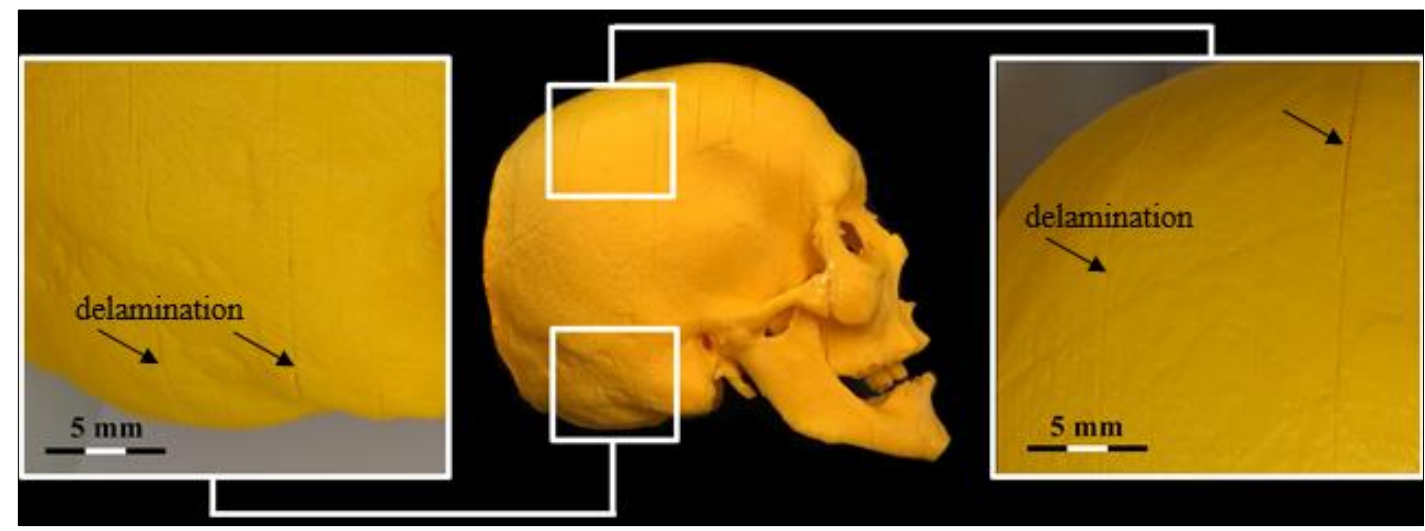

Figure 9. 3D printed skull at 1:1 scale

\subsection{Facial reconstruction}

Figure 10 presents the comparison between a skull made by manual casting process and the 3D printed skull based on the CT scan model. As seen in Figure 10a, the cast skull lacks certain features and details of the original skull. In contrast, the 3D printed skull accurately presents the anatomical details, such as cavities of the eyes and brain, the hollows of the denture. However, the surface of the cast skull is superior to the 3D printed skull.

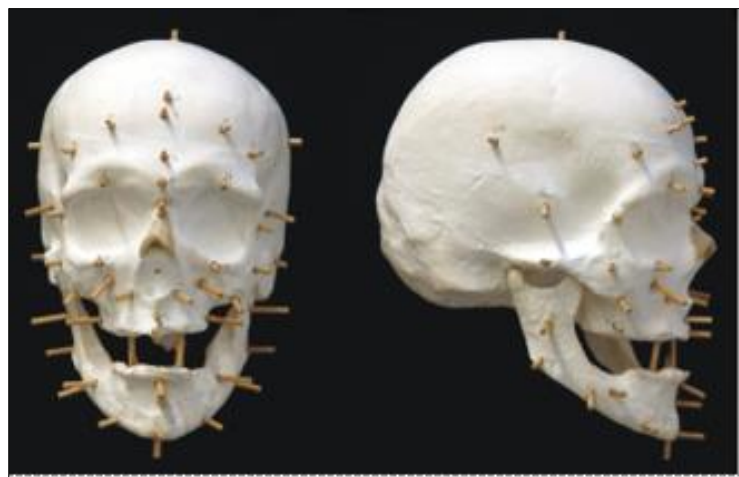

a) skull replica by manual casting process

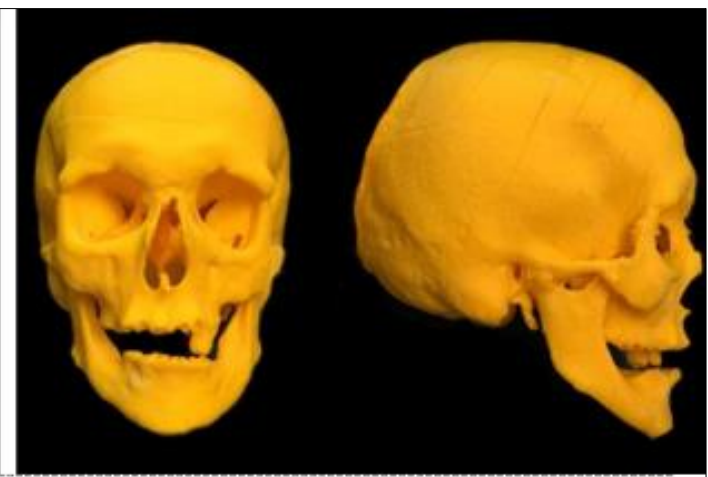

b) skull replica by $3 \mathrm{D}$ printing process

Figure 10. Replicas of the skull at 1:1 scale 
Figure 11 presents the facial reconstruction process on the 3D printed skull using the Manchester method [15, 16]. In this method, both facial muscles and soft tissue thickness are taken into consideration. The osteometric markers (landmarks indicating the soft tissue depth of the face) are added on the replica by inserting them on previously drilled holes [15]. The muscles of mastication and facial expression are then constructed out of the modeling material and prosthetic plastic eye balls are placed into the orbits [15]. The profile and shape of the nose are determined by the nasal aperture and the thickness of lips is determined by the anterior teeth [15]. The length of the markers is kept as a guide to the final tissue guides over the face [15].

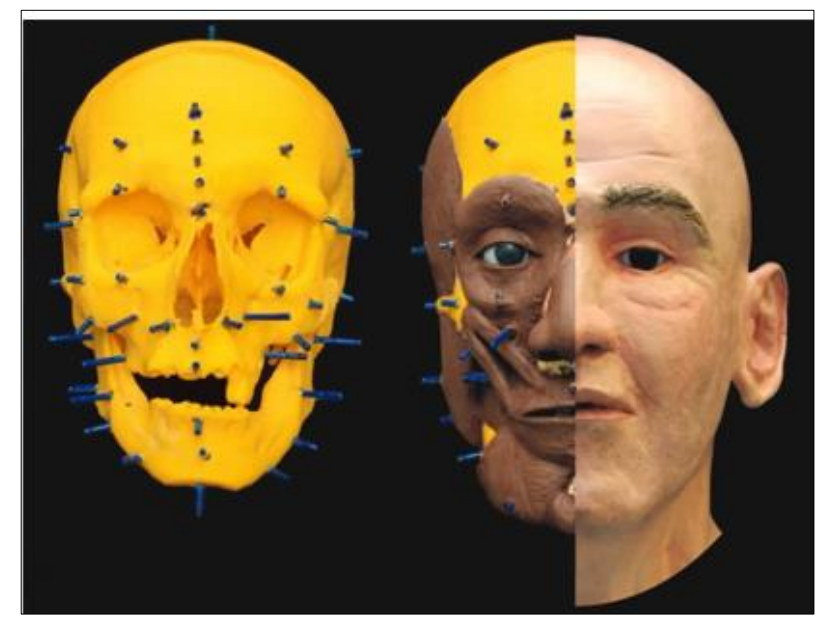

Figure 11. The facial reconstruction performed on the $3 \mathrm{D}$ printed skull

The skull is important forensic evidence and, in certain cases, can be fragile, thus the CT scan (followed by 3D printing a replica) can be the optimal strategy in order to perform a forensic facial reconstruction.

\section{Conclusions}

The current study presented the application of 3D printing in the forensic field in order to perform facial reconstruction on a 3D printed replica of the victim's skull. The 3D skull model was sliced and printed using an ABS filament on an in-house design 3D printer. To optimize the 3D printing parameters, the rheological behavior of the ABS was investigated using melt flow rate and capillary rheometry. The following main conclusions are drawn:

(i) The position of the 3D model in the slicing software is an important parameter to consider when printing complex parts. The face-upwards position of the skull model was found to be the optimal position since a larger contact surface between the 3D model and the building platform is needed.

(ii) The quality of the $3 \mathrm{D}$ printed skull is closely related to the quality of the CT scan and printing parameters, including layer thickness, and rheological properties of the ABS.

(iii) The optimal printing temperature was found to be $245^{\circ} \mathrm{C}$. Moreover, ABS shows shear-thinning behavior $(\mathrm{n}=0.312)$ and low activation energy (values between 3.899 to $2.697 \mathrm{~kJ} / \mathrm{mol}$ in the $50-100 \mathrm{~s}^{-1}$ range), which are important parameter for 3D printability, but not provided in the data sheet of the commercially available ABS filament.

By using 3D printing technology coupled with CT scan, skulls for facial reconstruction can be accurately reproduced, preserving the forensic evidence.

Acknowledgments: The work of Stanciu Nicoleta-Violeta was supported by the project ANTREPRENORDOC, within the framework of Human Resources Development Operational 
Programme 2014-2020, financed from the European Social Fund under contract number 36355/23.05.2019 HRD OP /380/6/13 - SMIS Code: 123847.

\section{References}

1. WILKINSON, C., Computerized Forensic Facial Reconstruction, Forensic Sci. Med. Pathol., 1, 2005, 173-177, https://doi.org/10.1385/FSMP:1:3:173.

2. FERNANDES, C.M.S., da COSTA SERRA, M., da SILVA, J.V.L, NORITOMI, P.Y., da SENA PEREIRA, D.A., HALTENHOFF MELANI, R.F., Tests of One Brazilian Facial Reconstruction Method Using Three Soft Tissue Depth Sets and Familiar Assessors, Forensic Sci. Int., 214, 2012, 211.e1211.e7, https://doi.org/10.1016/j.forsciint.2011.08.017.

3. FERNANDES, C.M.S., de SENA PEREIRA, F.D.A., da SILVA, J.V.L, da COSTA SERRA, M., Is Characterizing the Digital Forensic Facial Reconstruction with Hair Necessary? A Familiar Assessors' Analysis, Forensic Sci. Int., 229, 2013, 164. e1-164.e5, https://doi.org/10.1016/j.forsciint.2013.03.036. 4. SIMPSON, E., HENNEBERG, M., Variation in soft-tissue thicknesses on the human face and their relation to craniometric dimensions, Am. J. Phys. Anthropol., 118, 2002, 121-133,

https://doi.org/10.1002/ajpa.10073.

5. MARIC, J., BASIC, Z., JERKOVIC, I., MIHANOVIC, F., ANDELINOVIC, S., KRUZIC, I., Facial Reconstruction of Mummified Remains of Christian Saint-Nicolosa Bursa, J. Cult. Herit., 42, 2020, 249-254, https://doi.org/10.1016/j.culher.2019.08.008.

6. VERZE, L., History of Facial Reconstruction, Acta Biomed., 80(1), 2009, 5-12.

7. STAN, F., STANCIU, N.V., SANDU, I.L., FETECAU, C., SERBAN, A., Effect of Low and ExtremeLow Temperature on Mechanical Properties of 3D-Printed Polyethylene Terephthalate Glycol, Ro. $J$. Techn. Sci. - Appl. Mechanics, 64, 2019, 21-41.

8. STAN F., STANCIU N.V., FETECAU C., On the 3D Printability of Multi-Walled Carbon Nanotube/High Density Polyethylene Composites, Proceeding of the ASME 2019 14th International Manufacturing Science and Engineering Conference, MSEC2019-2776, V002T04A004, 10 pages, https://doi.org/10.1115/MSEC2019-2776.

9. STANCIU, N.V., STAN, F., FETECĂU, C., SUSAC, F., On the Feasibility of Printing 3D Composite Objects Based on Polypropylene/Multi-Walled Carbon Nanotubes, 9th International Conference on Manufacturing Science and Education (MSE 2019), 03017, 14 pages,

https://doi.org/10.1051/matecconf/201929003017.

10. STAN F., STANCIU N.V., CONSTANTINESCU, M., FETECAU, C., 3D Printing of Flexible and Stretchable Parts Using Multiwall Carbon Nanotubes/polyester-Based Thermoplastic Polyurethane, $J$. Manuf. Sci. Eng., 2020, 1-33, https://doi.org/10.1115/1.4048442.

11. YAN, Q., DOONG, H., SU, J., HAN, J., SONG, B., WEI, Q., SHI, Y., A Review of 3D Printing Technology for Medical Applications, Engineering, 4, 2018, 729-742,

https://doi.org/10.1016/j.eng.2018.07.021.

12. NGO, T.D., KASHANI, A., IMBALZANO, G., NGUYEN, K.T.Q., HUI, D., Additive Manufacturing (3D Printing): A Review of Materials, Methods, Applications and Challenges, Compos. Part B, 143, 2018, 172-196, https://doi.org/10.1016/j.compositesb.2018.02.012.

13. SHEN, Z., YAO, Y., XIE, Y., GUO, C., SHANG, X., DONG, X., LI, Y., PAN, Z., CHEN, S., XIONG, G., WANG, F.Y., PAN, H., The Process of 3D Printed Skull Models for Anatomy Education, Computer. Assist. Surg., 24, 2019, https://doi.org/10.1080/24699322.2018.1560101.

14. BARTELLAS, M., Three-Dimensional Printing and Medical Education: A Narrative Review of the Literature, University of Ottawa Journal of Medicine, 6, 2016, 38,

https://doi.org/10.18192/uojm.v6i1.1515.

15. GUPTA. S., GUPTA, V., VIJ, H., VIJ, R., TYAGI, N., Forensic Facial Reconstruction: The Final Frontier, J. Clin. Diagn. Res., 9(9), 2015, ZE26-ZE28, https://doi:10.7860/JCDR/2015/14621.6568. 16. WILKINSON, C. The Manchester method of facial reconstruction. In Forensic Facial Reconstruction, Cambridge: Cambridge University Press, 2004, 157-199. 
17. Bringing Closure to Families. 3D Printing and Facial Reconstruction Help Identify Eight Victims from US-Mexico Border. Available online: https://all3dp.com/3d-printing-facial-reconstruction-helpidentify-eight-victims-us-mexico-border (accessed on 19 June 2020).

18. MORRISON, F.A., Understanding rheology, Oxford University Press, Inc., New York, 2001, 454.

19. Product Data Sheet: ABS Filament, Verbatim.

20. 3D Printing Filament for Beginners

ABS Filament for 3D Printing - All You Need to Know. Available online: https://all3dp.com/abs-3dprinter-filament-explained/

21. KUMAR, N., JAIN, P.K., TANDON, P., Investigations on the Melt Flow Behaviour of Aluminium Filled ABS Polymer Composite for the Extrusion-Based Additive Manufacturing Process, Int. J. Mater. Prod. Tec., 59, 2019, 194-211.

Manuscript received: 6.08 .2020 